\title{
ELECTROMAGNETIC WAVES IN A BENT PIPE OF RECTANGULAR CROSS SECTION*
}

\author{
BY \\ KARLEM RIESS \\ Tulane University
}

The analysis of electromagnetic wave propagation in a bent pipe of rectangular cross section, $(x=R, x=R+a, y=0, y=b)$, is based on the Maxwell field equations, expressed in cylindrical coordinates $(r, \theta, y)$ (Fig. 1). As in the case of the straight pipe, ${ }^{1}$ the time variation is given by the exponential $e^{j \omega t}$, where $\omega$ is the angular frequency. The angular variation is given by $e^{-\Sigma \theta}$, where $\Sigma$ is the propagation constant for the bent portion. The equations may be written

$$
\begin{array}{r}
-\Sigma E_{y}-r \partial E_{\theta} / \partial y+r j \omega \mu H_{r}=0 \\
\partial E_{r} / \partial y-\partial E_{y} / \partial r+j \omega \mu H_{\theta}=0 \\
r \partial E_{\theta} / \partial r+E_{\theta}+\Sigma E_{r}+r j \omega \mu H_{y}=0 \\
-\Sigma H_{y}-r \partial H_{\theta} / \partial y-r j \omega \epsilon E_{r}=0 \\
\partial H_{r} / \partial y-\partial H_{y} / \partial r-j \omega \epsilon E_{\theta}=0 \\
r \partial H_{\theta} / \partial r+H_{\theta}+\Sigma H_{r}-r j \omega \epsilon E_{y}=0 \\
r \partial H_{r} / \partial r+H_{r}-\Sigma H_{\theta}+r \partial H_{\nu} / \partial y=0 \\
r \partial E_{r} / \partial r+E_{r}-\Sigma E_{\theta}+r \partial E_{y} / \partial y=0 .
\end{array}
$$

In (1) $H_{\theta}, H_{r}, H_{y}, E_{\theta}, E_{r}$ and $E_{y}$ are the components of magnetic and electric field, $\epsilon$ is the electric inductive capacity, and $\mu$ the magnetic inductive capacity. The electrical conductivity, $\sigma$, and charge density, $\rho$, are assumed to be zero.

The field components $H_{r}, H_{y}, E_{r}$ and $E_{y}$ may be expressed in terms of $H_{\theta}$ and $E_{\theta}$ by various combinations of the equations (1). These give

where

$$
\begin{aligned}
H_{r}\left(G r^{2}\right) & =-\Sigma r \partial H_{\theta} / \partial r-\Sigma H_{\theta}-r^{2} j \omega \epsilon \partial E_{\theta} / \partial y, \\
H_{y}\left(G r^{2}\right) & =-\Sigma r \partial H_{\theta} / \partial y+r j \omega \epsilon E_{\theta}+r^{2} j \omega \epsilon \partial E_{\theta} / \partial r, \\
E_{r}\left(G r^{2}\right) & =-\Sigma r \partial E_{\theta} / \partial r-\Sigma E_{\theta}+r^{2} j \omega \mu \partial H_{\theta} / \partial y, \\
E_{\nu}\left(G r^{2}\right) & =-\Sigma r \partial E_{\theta} / \partial y-r j \omega \mu H_{\theta}-r^{2} j \omega \mu \partial H_{\theta} / \partial r,
\end{aligned}
$$

$$
\left(G r^{2}\right)=\Sigma^{2}+r^{2} \omega^{2} \mu \epsilon \text {. }
$$

Using the last two of Eqs. (1) and Eqs. (2), $H_{r}, H_{y}, E_{r}$ and $E_{y}$ may be eliminated and equations in $H_{\theta}$ and $E_{\theta}$ readily obtained.

$$
\frac{\partial}{\partial r}\left[\frac{1}{G r} \frac{\partial\left(r H_{\theta}\right)}{\partial r}\right]+H_{\theta}+G^{-1} \frac{\partial^{2} H_{\theta}}{\partial y^{2}}+\frac{r j \omega \epsilon}{\Sigma} \frac{\partial E_{\theta}}{\partial y} \frac{\partial G^{-1}}{\partial r}=0,
$$

* Received June 29, 1943. The paper constitutes part of a thesis submitted in partial fulfillment of the requirements for the degree of Doctor of Philosophy at Brown University, May, 1943. The author wishes to thank Professor L. Brillouin of Brown University for his many helpful suggestions.

1 This case has been discussed by Lord Rayleigh, Phil. Mag. 43, 125-132 (1897); Brillouin, Rev. Gén. de l'Elec. 40, 227-239 (1936); Schelkunoff, Proc. Inst. Radio Eng. 25, 1457-1492 (1937); Chu and Barrow, Proc. Inst. Radio Eng. 26, 1520-1555 (1938); Slater, Microwave transmission, McGraw-Hill, 1942, pp. 124-150. 


$$
\frac{\partial}{\partial r}\left[\frac{1}{G r} \frac{\partial\left(r E_{\theta}\right)}{\partial r}\right]+E_{\theta}+G^{-1} \frac{\partial^{2} E_{\theta}}{\partial y^{2}}-\frac{r j \omega \mu}{\Sigma} \frac{\partial H_{\theta}}{\partial y} \frac{\partial G^{-1}}{\partial r}=0 .
$$

The boundary conditions for this case are

$$
\begin{array}{lll}
y=0, & y=b . & E_{\theta}=0, \quad E_{r}=0 . \\
r=R, & r=R+a . & E_{\theta}=0, \quad E_{y}=0 .
\end{array}
$$

A more useful form may be obtained from (2d):

$$
\begin{aligned}
& y=0, \quad y=b . \quad E_{\theta}=0, \quad E_{r}=0 . \\
& r=R, \quad r=R+a . \quad E_{\theta}=0, \quad \partial\left(r H_{\theta}\right) / \partial r=0 .
\end{aligned}
$$

By considering $E_{\theta}$ and $H_{\theta}$ as functions of $y$ and $r$, these conditions establish the dependence of $E_{\theta}$ on $\sin k_{y} y$, and $H_{\theta}$ on $\cos k_{y} y$, where $k_{y}=n \pi / b, n$ being an integer. By substituting

$$
E_{\theta}(r, y)=E_{\theta}(r) \sin k_{y} y, \quad H_{\theta}(r, y)=H_{\theta}(r) \cos k_{y} y,
$$

in (3) and simplifying, equations in $E_{\theta}$ and $H_{\theta}$ as functions of $r$ alone are obtained.

$$
\begin{aligned}
& \frac{\partial^{2} H_{\theta}}{\partial r^{2}}+A(r) \frac{\partial H_{\theta}}{\partial r}+B(r) H_{\theta}+\epsilon C(r) E_{\theta}=0, \\
& \frac{\partial^{2} E_{\theta}}{\partial r^{2}}+A(r) \frac{\partial E_{\theta}}{\partial r}+B(r) E_{\theta}+\mu C(r) H_{\theta}=0,
\end{aligned}
$$

where the coefficients $A(r), B(r)$ and $C(r)$ have the values

$$
\begin{aligned}
& A(r)=\frac{1}{r}+\frac{2 \Sigma^{2}}{G r^{3}}=\frac{1}{r}+\frac{2 \Sigma^{2}}{r\left(\Sigma^{2}+r^{2} \omega^{2} \mu \epsilon\right)}, \\
& B(r)=G-k_{y}^{2}-\frac{1}{r^{2}}+\frac{2 \Sigma^{2}}{G r^{4}}=\frac{\Sigma^{2}-1}{r^{2}}+\omega^{2} \mu \epsilon-k_{y}^{2}+\frac{2 \Sigma^{2}}{r^{2}\left(\Sigma^{2}+r^{2} \omega^{2} \mu \epsilon\right)}, \\
& C(r)=\frac{2 j k_{y} \omega \Sigma}{G r^{2}}=\frac{2 j k_{y} \omega \Sigma}{\left(\Sigma^{2}+r^{2} \omega^{2} \mu \epsilon\right)} .
\end{aligned}
$$

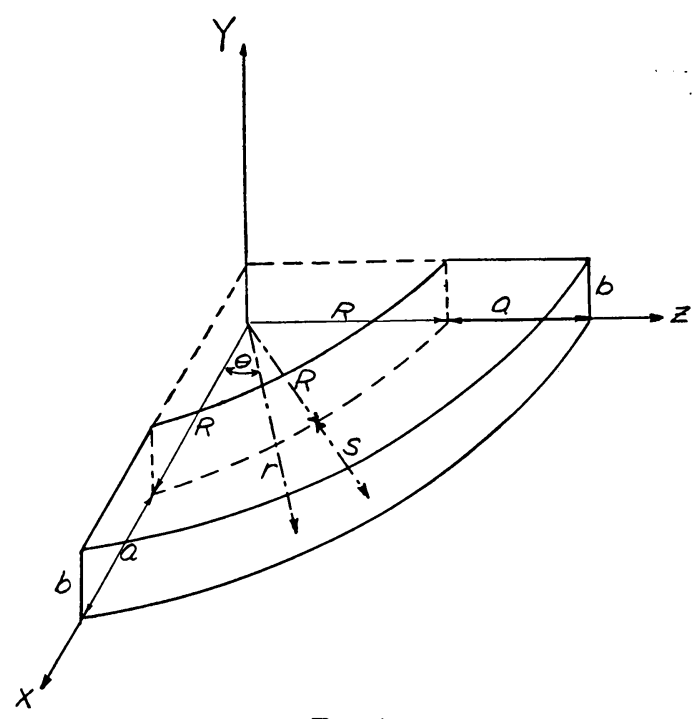

FIG. 1

Equations (6a) and (6b) show that $\bar{E}_{\theta}$ and $H_{\theta}$ are not independent in the bent pipe. Furthermore, $E_{\theta}$ and $H_{\theta}$ do not vanish in this case, hence the methods of solution used for the straight pipe fail. $E_{\theta}$ and $H_{\theta}$ are not expressible in terms of Bessel functions. One possible method of solution of these equations, namely to substitute

$$
\begin{aligned}
& \phi_{1}=(\mu)^{1 / 2} H_{\theta}+(\epsilon)^{1 / 2} E_{\theta}, \\
& \phi_{2}=(\mu)^{1 / 2} H_{\theta}-(\epsilon)^{1 / 2} E_{\theta},
\end{aligned}
$$

and thus to obtain separated equations in $\phi_{1}$ and $\phi_{2}$, is incorrect because the boundary conditions (4) are not satisfied.

Equations (6a) and (6b) have been 
solved completely by a method of approximation, using the theory of the Schrödinger equation with perturbations. Only the zero order and first order terms are considered. This does not affect the generality of the solution, because in practice the radius of curvature of the pipe, $R$, may be chosen very large compared to the constants of the equations and to the dimension $a$ of the pipe.

To rewrite (6a) and (6b) in the familiar Schrödinger form, let.

$$
\begin{gathered}
\left(r H_{\theta}\right) / R=\beta, \\
r=R+s=R(1+s / R), \quad 0<s<a, \\
\gamma=\Sigma / R .
\end{gathered}
$$

Thus

$$
\begin{aligned}
\partial^{2} \beta / \partial s^{2}+f_{1}(s) \partial \beta / \partial s+g(s) \beta+h(s) \epsilon E_{\theta} & =0, \\
\partial^{2} E_{\theta} / \partial s^{2}+f_{2}(s) \partial E_{\theta} / \partial s+g(s) E_{\theta}+h(s) \mu H_{\theta} & =0 .
\end{aligned}
$$

The coefficients, to the first approximation in $R^{-1}$, are given by

where

$$
\begin{aligned}
f_{1}(s)= & R^{-1}\left(-1+2 \gamma^{2} / K^{2}\right), \quad f_{2}(s)=R^{-1}\left(1+2 \gamma^{2} / K^{2}\right), \\
& g(s)=K^{2}-k_{\nu}^{2}-2 \gamma^{2} s / R=k_{x}^{2}-2 \gamma^{2} s / R, \\
& h(s)=2 j k_{y} \omega \gamma / R K^{2},
\end{aligned}
$$

$$
K^{2}=\gamma^{2}+\omega^{2} \mu \epsilon=k_{x}^{2}+k_{y}^{2} .
$$

Continuing the approximation, $E_{\theta}$ and $\beta$ may be written as

$$
E_{\theta}=\left(E_{\theta}\right)_{0}+R^{-1}\left(E_{\theta}\right)_{1}+\cdots, \quad \beta=\beta_{0}+R^{-1} \beta_{1}+\cdots,
$$

and the perturbation of the angular coefficient for each case as

$$
k_{x}^{2}=k_{s}^{2}+R^{-1} e_{1} \quad \text { for } E_{\theta}, \quad k_{x}^{2}=k_{s}^{2}+R^{-1} h_{1} \text { for } \beta .
$$

By substituting (10) and (11) in (9), the zero order and first order approximations may be written separately. For $E_{\theta}$ these are

$$
\begin{gathered}
\frac{\partial^{2}\left(E_{\theta}\right)_{0}}{\partial s^{2}}+k_{s}^{2}\left(E_{\theta}\right)_{0}=0, \\
\frac{\partial^{2}\left(E_{\theta}\right)_{1}}{\partial s^{2}}+\frac{\partial\left(E_{\theta}\right)_{0}}{\partial s}\left[1+2 \gamma^{2} / K^{2}\right]+\left(E_{\theta}\right)_{1} k_{s}^{2} \\
+e_{1}\left(E_{\theta}\right)_{0}-2 \gamma^{2} s\left(E_{\theta}\right)_{0}+\frac{2 j k_{y} \gamma \omega \mu\left(H_{\theta}\right)_{0}}{R K^{2}}=0 .
\end{gathered}
$$

The zero order equation (12a) has the same form as the equation for the straight pipe, with the solution

$$
\left(E_{\theta}\right)_{0}=E_{m, n} \sin k_{s} s,
$$

where $m$ and $n$ are integers.

Similar equations may be written for $\beta$, giving

$$
\beta_{0}=\lim \frac{r}{R}\left(H_{\theta}\right)_{0}=H_{m, n} \cos k_{8} s=\left(H_{\theta}\right)_{0} .
$$


Eq. (12b) may be rewritten as

$$
\begin{aligned}
\frac{\partial^{2}\left(E_{\theta}\right)_{1}}{\partial s^{2}}+k_{s}^{2}\left(E_{\theta}\right)_{1}= & -e_{1}\left(E_{\theta}\right)_{0}+2 \gamma^{2} s\left(E_{\theta}\right)_{0} \\
& -\left(1+2 \gamma^{2} / K^{2}\right) \frac{\partial\left(E_{\theta}\right)_{0}}{\partial s}-\frac{2 j k_{y} \omega \mu \gamma\left(H_{\theta}\right)_{0}}{K^{2}} .
\end{aligned}
$$

This is the general form of the Schrödinger equation with perturbations, where the usual perturbation factor $\lambda$ is equal to $1 / R$.

By using the orthogonality condition for the Schrödinger theory, the value of $e_{1}$ may be readily determined:

$$
\begin{aligned}
\int_{0}^{a}\left(-e_{1}+2 \gamma^{2} s\right) E_{m, n}^{2} \sin ^{2} k_{s} s d s & -\int_{0}^{a}\left(1+2 \gamma^{2} / K^{2}\right) k_{s} E_{m, n}^{2} \cos k_{s} s \sin k_{s} s d s \\
& -\int_{0}^{a} 2 j k_{\imath} \omega \mu \gamma K^{-2} H_{m, n} E_{m, n} \cos k_{s} s \sin k_{s} s d s=0 .
\end{aligned}
$$

Therefore

$$
e_{1}=\gamma^{2} a \text {. }
$$

By using this value and (13), the first approximation (15) may be solved for $\left(E_{\theta}\right)_{1}$. The solution, satisfying the boundary conditions, is given by

$$
\begin{aligned}
\left(E_{\theta}\right)_{1}= & E_{m, n} \cos k_{s} s\left[\left(\gamma^{2} s\right)(a-s)\left(2 k_{8}\right)^{-1}\right] \\
& +E_{m, n} \sin k_{s} s\left[(s)\left(2 k_{s}^{2}\right)^{-1}\left\{\gamma^{2}-\left[1+\frac{2 \gamma^{2}}{K^{2}}\right] k_{s}^{2}\right\}\right] \\
& -H_{m, n} \sin k_{s} s\left[\left(j k_{y} s \omega \mu \gamma\right)\left(K^{2} k_{8}\right)^{-1}\right] .
\end{aligned}
$$

In like manner, from the $\beta$ approximation equations,

$$
h_{1}=\gamma^{2} a \text {. }
$$

Since $e_{1}=h_{1}$, there is no change in the angle variable during the perturbations. The solution of the $\beta$ equation, satisfying the boundary conditions, and corresponding to (16a) is

$$
\begin{aligned}
\beta_{1}= & E_{m, n} \cos k_{8} s\left[\left(j k_{y} s \omega \epsilon \gamma\right)\left(k_{8} K^{2}\right)^{-1}\right]-E_{m, n} \sin k_{8} s\left[\left(j k_{y} \omega \epsilon \gamma\right)\left(k_{8}^{2} K^{2}\right)^{-1}\right] \\
& +H_{m, n} \cos k_{s} s\left[(s / 2)\left(1-2 \gamma^{2} / K^{2}+\gamma^{2} / k_{8}^{2}\right)\right] \\
& +H_{m, n} \sin k_{s} s\left[\left(2 k_{8}\right)^{-1}\left(-\gamma^{2} a s-1+\gamma^{2} s^{2}+2 \gamma^{2} / K^{2}-\gamma^{2} / k_{s}^{2}\right)\right] .
\end{aligned}
$$

The complete solutions of (6a) and (6b), including both the zero order and first order approximations, may be written as

$$
\begin{aligned}
E_{\theta}= & \left\{E_{m, n} \sin k_{s} s\left[1+c_{1} s\right]+E_{m, n} \cos k_{s} s\left[s(a-s) c_{2}\right]\right. \\
& \left.-H_{m, n} \sin k_{s} s\left[\mu c_{3} s\right]\right\}\left\{\sin k_{y} y e^{j \omega t-\Sigma \theta}\right\}, \\
H_{\theta}= & \left\{H_{m, n} \cos k_{s} s\left[1+c_{1} s\right]-H_{m, n} \sin k_{s} s\left[s(a-s) c_{2}-c_{4}\right]\right. \\
& \left.-E_{m, n} \sin k_{s} s\left[\epsilon c_{3} / k_{s}\right]+E_{m, n} \cos k_{s} s\left[\epsilon c_{3} s\right]\right\}\left\{\cos k_{y} y e^{j \omega t-\Sigma \theta}\right\},
\end{aligned}
$$


where

$$
\begin{aligned}
& c_{1}=\frac{\gamma^{2}}{2 R k_{s}^{2}}-\frac{1}{2 R}-\frac{\gamma^{2}}{R K^{2}}, \quad c_{2}=\frac{\gamma^{2}}{2 R k_{s}}, \\
& c_{3}=\frac{j k_{y} \omega \gamma}{R k_{8} K^{2}}, \quad c_{4}=\frac{-\gamma^{2}}{2 R k_{s}^{3}}-\frac{1}{2 R k_{s}}+\frac{\gamma^{2}}{R K^{2} k_{s}} .
\end{aligned}
$$

By using (2) and the approximations (8) and (10), the components $H_{r}, H_{y}, E_{r}$ and $E_{y}$ are seen to become

$$
\begin{aligned}
&-K^{2} H_{r}=\left\{H _ { m , n } \operatorname { s i n } k _ { s } s \left[-\gamma k_{s}\left(1+c_{1} s-s / R+2 \gamma^{2} s / R K^{2}\right)\right.\right. \\
&\left.+c_{2}(2 s-a) \gamma-j \omega \mu \epsilon c_{3} k_{y} s\right] \\
&+H_{m, n} \cos k_{s} s\left[\gamma\left\{c_{1}+R^{-1}-c_{2} s(a-s) k_{s}\right\}+\gamma k_{s} c_{4}\right] \\
&+E_{m, n} \sin k_{s} s\left[j \omega \epsilon k_{y}\left(1+c_{1} s+2 \gamma^{2} s / R K^{2}\right)-k_{s} c_{3} \gamma \epsilon s\right] \\
&\left.+E_{m, n} \cos k_{s} s\left[j \omega \epsilon k_{y} s(a-s) c_{2}\right]\right\} \cos k_{y} y e^{j \omega t-\Sigma \theta} \\
& K^{2} H_{y}=\left\{E_{m, n} \sin k_{s} s\left[j \omega \epsilon c_{1}-k_{s} s(a-s) c_{2}+R^{-1}\right\}-\left(c_{3} k_{y} \gamma \epsilon\right)\left(k_{s}\right)^{-1}\right] \\
&+E_{m, n} \cos k_{s} s\left[j \omega \epsilon k_{s}\left(1+c_{1} s+2 \gamma^{2} s / R K^{2}\right)-j \omega \epsilon(2 s-a) c_{2}+k_{y} c_{3} \gamma \epsilon s\right] \\
&-H_{m, n} \sin k_{s} s\left[j \omega \mu \epsilon c_{3}+\gamma k_{y}\left\{s(a-s) c_{2}-c_{4}\right\}\right] \\
&\left.+H_{m, n} \cos k_{s} s\left[-j \omega \mu \epsilon k_{s} c_{3} s+\gamma k_{y}\left(1+c_{1} s-s / R+2 \gamma^{2} s / R K^{2}\right)\right]\right\} \\
& \cdot \sin k_{y} y e^{j \omega t-\Sigma \theta}, \\
&-K^{2} E_{r}=\left\{E_{m, n} \sin k_{s} s\left[\gamma\left\{c_{1}-c_{2} s(a-s) k_{s}+R^{-1}\right\}-\left(k_{y} c_{3} j \omega \mu \epsilon\right)\left(k_{s}\right)^{-1}\right]\right. \\
&+E_{m, n} \cos k_{s} s\left[\gamma k_{s}\left(1+c_{1} s-s / R+2 \gamma^{2} s / R K^{2}\right)+j \omega \mu \epsilon k_{y} c_{3} s-c_{2} \gamma(2 s-a)\right] \\
&-H_{m, n} \sin k_{s} s\left[c_{3} \gamma \mu+j \omega \mu k_{y}\left\{s(a-s) c_{2}-c_{4}\right\}\right] \\
&\left.+H_{m, n} \cos k_{s} s\left[j \omega \mu k_{y}\left(1+c_{1} s+2 \gamma^{2} s / R K^{2}\right)-k_{s} c_{3} \gamma \mu s\right]\right\} \\
& \cdot \sin k_{y} y e^{j \omega t-\Sigma \theta} \\
&+\cos k_{y} y e^{j \omega t-\Sigma \theta} . \\
&+E_{m, n} \sin k_{s} s\left[\gamma k_{y}\left(1+c_{1} s-s / R+2 \gamma^{2} s / R K^{2}\right)-c_{3} k_{s} j \omega \mu \epsilon s\right] \\
&+H_{m, n} \sin k_{s} s\left[-j \omega \mu k_{s}\left(1+c_{1} s+2 \gamma^{2} s / R K^{2}\right)+j \omega \mu c_{2}(2 s-a)-k_{y} c_{3} s \gamma \mu\right] \\
&\left.+H_{m, n} \cos k_{s} s\left[j \omega \mu\left\{c_{1}-k_{s} s(a-s) c_{2}+R^{-1}\right\}+j \omega \mu k_{s} c_{4}\right]\right\} \\
&-K^{2} E_{y} s(18 \mathrm{~d}) \\
& \\
&
\end{aligned}
$$

The solutions for the field components (18) satisfy the Maxwell field equations (1) within the approximation conditions imposed on the solution of the problem.

For the special cases of $H_{m, n}$ and $E_{m, n}$ when one of the integers $m$ or $n$ is zero, the components may be obtained from (18). For $m=0$ and $n$ not equal to zero:

$$
\begin{aligned}
E_{\theta} & =H_{0, n}\left[j \omega \mu \gamma k_{y} s(a-s) / R K_{m}^{2}\right] \sin k_{y} y e^{j \omega t-\Sigma \theta}, \\
H_{\theta} & =H_{0, n}\left[1-s / R-\gamma^{2} a s^{2} / 2 R+\gamma^{2} s^{3} / 3 R\right] \cos k_{y} y e^{j \omega t-\Sigma \theta}, \\
K_{m}^{2} H_{r} & =H_{0, n}\left[\gamma s(a-s) R^{-1}\left(\omega^{2} \mu \epsilon k_{y}^{2} / K_{m}^{2}+\gamma^{2}\right)\right] \cos k_{y} y e^{j \omega t-\Sigma \theta}, \\
K_{m}^{2} H_{y} & =H_{0, n}\left[k_{y} \gamma R^{-1}\left(-\omega^{2} \mu \epsilon a / K_{m}^{2}+R-\gamma^{2} a s^{2} / 2+\gamma^{2} s^{3} / 3\right)\right] \sin k_{y} y e^{j \omega t-\Sigma \theta}, \\
-K_{m}^{2} E_{r} & =H_{0, n}\left[j \omega \mu k_{y} R^{-1}\left(\gamma^{2} a / K_{m}^{2}+R-s-\gamma^{2} a s^{2} / 2+\gamma^{2} s^{3} / 3\right)\right] \sin k_{y} y e^{j \omega t-\Sigma \theta}, \\
E_{y} & =0,
\end{aligned}
$$


where

$$
K_{m}^{2}=\left(K^{2}\right)_{m=0}=k_{y}^{2}+\gamma^{2} a / R .
$$

For $m$ not equal to zero, $n=0$ :

$$
\begin{aligned}
E_{\theta}= & 0, \\
H_{\theta}= & \left\{H_{m, 0} \cos k_{s} s\left[1+c_{1} s\right]-H_{m, 0} \sin k_{8} s\left[(a-s) c_{2} s-c_{4}\right]\right\} \cos k_{y} y e^{j \omega t-\Sigma \theta}, \\
-K_{n}^{2} H_{r}= & \left\{H_{m, 0} \sin k_{s} s\left[c_{2} \gamma(2 s-a)-\gamma k_{s}\left(1+c_{1} s-s / R+2 \gamma^{2} s / R K_{n}^{2}\right)\right]\right. \\
& \left.+H_{m, 0} \cos k_{s} s\left[\gamma\left\{c_{1}+R^{-1}-c_{2} s(a-s) k_{s}\right\}+k_{s} c_{4} \gamma\right]\right\} \cos k_{y} y e^{j \omega t-\Sigma \theta}, \\
H_{y}= & 0, \quad E_{r}=0, \\
-K_{n}^{2} E_{y}= & \left\{H_{m, 0} \sin k_{s} s\left[-j \omega \mu k_{8}\left(1+c_{1} s+2 \gamma^{2} s / R K_{n}^{2}\right)+j \omega \mu c_{2}(2 s-a)\right]\right. \\
& +H_{m, 0} \cos k_{s} s\left[j \omega \mu\left\{c_{1}-k_{8} s(a-s) c_{2}+R^{-1}\right\}+j \omega \mu c_{4} k_{s}\right] \cos k_{y} y e^{j \omega t-\Sigma \theta},
\end{aligned}
$$

where the $c_{1}, c_{2}, c_{3}, c_{4}$ are calculated for $n$ vanishing, and

$$
K_{n}^{2}=\left(K^{2}\right)_{n=0}=k_{s}^{2}+\gamma^{2} a / R .
$$

It should be noted that both the $E_{0, n}$ and $E_{m, 0}$ are missing.

A consideration of the continued propagation of $E$ and $H$ waves from a straight pipe into a bent pipe yields some interesting results. A pure $E_{m, n}$ or $H_{m, n}$ wave in the straight pipe will be reflected, partially, at the junction with the bent pipe. After reflection the amplitudes are proportional to $a / R$, and intensities to $a^{2} / R^{2}$, hence, for the first approximation, the reflected portion may be neglected. Thus a pure $E_{m, n}$ or a pure $H_{m, n}$ wave in the straight pipe may be traced into the bent pipe, where it will become a mixed $E$ and $H$ wave.

For a mixed $E$ and $H$ wave in the straight portion, the intensities are proportional to $a / R$ and must be considered. A mixed $E$ and $H$ wave in the straight pipe, because of the reflected portion at the junction, sets up an undetermined condition within the pipe, not predictable from the results of this paper.

If the propagation constant is measured along the center line, $a / 2$, of the bent pipe, there is no change in its value from that of the straight pipe. 